\title{
On Tabor groupoids and stability of some functional equations
}

\author{
Roman Badora, Barbara Przebieracz and Peter Volkmann
}

\begin{abstract}
Two results are given, which use Tabor groupoids for questions of stability in the sense of Pólya-Szegö-Hyers-Ulam. We also start to study Tabor groupoids in their own right.
\end{abstract}

Mathematics Subject Classification (2010). 20N02, 39B82.

Keywords. Tabor groupoids, stability of functional equations, bounded perturbations of additive functions.

\section{Introduction}

We use the notations $N=\{1,2,3, \ldots\}, N_{0}=N \cup\{0\}$, and $R$ for the space of the reals.

Let $S$ be a groupoid, i.e., $S$ is a set and for all $x, y \in S$ we have a product $x y \in S$. For $x \in S$ and $k \in N_{0}$ the powers $x^{2^{k}}$ are recursively defined by

$$
x^{2^{0}}=x^{1}=x, \quad x^{2^{k+1}}=x^{2^{k}} x^{2^{k}} .
$$

Definition. A groupoid $S$ is called a Tabor groupoid, if the following condition holds:

(T) For $x, y \in S$ there always exists $k \in N$ such that

$$
(x y)^{2^{k}}=x^{2^{k}} y^{2^{k}} .
$$

This notion had been introduced in [1] on the basis of Józef Tabor's Remark [9]. Examples are groupoids $S$ with a square-symmetric operation, i.e.

$$
(x y)^{2}=x^{2} y^{2} \quad(x, y \in S) .
$$

As a consequence of [11] (cf. also Theorem 1 in [1]) we have the following result: 
Theorem 1. Let $S$ be a Tabor groupoid, and let $V$ be a bounded closed convex subset of a Banach space $E$. For $f: S \rightarrow E$ we suppose

$$
f(x y)-f(x)-f(y) \in V \quad(x, y \in S) .
$$

Then there exists a (unique) additive function $a: S \rightarrow E$ such that $a(x)-$ $f(x) \in V \quad(x \in S)$.

Here the additivity of $a: S \rightarrow E$ means

$$
a(x y)=a(x)+a(y) \quad(x, y \in S) .
$$

In the next Section we shall use Theorem 1 to characterize bounded perturbations of additive functions. In Section 3 we examine semigroups $S$ containing an idempotent element $s$ (i.e., $s^{2}=s$ ). We give sufficient conditions for them to be Tabor groupoids. We also show that the symmetric group $S_{3}$ is not a Tabor groupoid.

In Section 4 we study the functional equation

$$
\max \{f((x y) y), f(x)\}=f(x y)+f(y) \quad(x, y \in S)
$$

for functions $f: S \rightarrow R$, where $S$ is a groupoid having a left unit and satisfying the following condition:

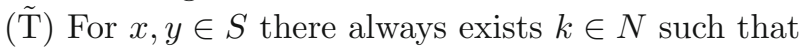

$$
(x y)^{2^{k}}=x^{2^{k}} y^{2^{k}}, \quad((x y) y)^{2^{k}}=\left(x^{2^{k}} y^{2^{k}}\right) y^{2^{k}} .
$$

$((\tilde{\mathrm{T}}) \Rightarrow(\mathrm{T})$, hence $S$ is a Tabor groupoid.) We prove the stability of (1.4), which in the special case (1.2) of $(\tilde{\mathrm{T}})$ goes back to Attila Gilányi, Kaori Nagatou (長藤かおり) and Volkmann [4]. In the case of a commutative group $(S,+)$, equation $(1.4)$ is equivalent to

$$
\max \{f(x+y), f(x-y)\}=f(x)+f(y) \quad(x, y \in S),
$$

the solutions of which are $f(x)=|a(x)|, a:(S,+) \rightarrow R$ being additive (Alice Simon and Volkmann [8]; cf. also the comments in [4]).

Finally, let us mention that in [2] we have shown the stability of the functional equation $f(x y)=f(y x)$ for $f: S \rightarrow E, S$ being a group and $E$ a normed space.

\section{A characterization of bounded perturbations of additive functions}

Theorem 2. Let $S$ be a Tabor groupoid, E a Banach space, $f: S \rightarrow E$, and suppose $A$ to be a bounded closed subset of $E$. Then the following two conditions are equivalent:

(P) $f=a+r$, where $a: S \rightarrow E$ is additive and $r(x) \in A(x \in S)$. 
(Q) There exist bounded subsets B, C of E such that

$$
\begin{gathered}
f(x y)-f(x)-f(y) \in B \quad(x, y \in S), \\
2^{k} f(x)-f\left(x^{2^{k}}\right) \in 2^{k} A+C \quad(x \in S, k \in N) .
\end{gathered}
$$

Proof. $(\mathrm{P}) \Rightarrow(\mathrm{Q})$ : From $(\mathrm{P})$ we get for $x, y \in S$ that

$$
\begin{aligned}
f(x y)-f(x)-f(y) & =r(x y)-r(x)-r(y) \in A-A-A, \\
2^{k} f(x)-f\left(x^{2^{k}}\right) & =2^{k} a(x)+2^{k} r(x)-a\left(x^{2^{k}}\right)-r\left(x^{2^{k}}\right) \\
& =2^{k} r(x)-r\left(x^{2^{k}}\right) \in 2^{k} A-A \quad(k \in N),
\end{aligned}
$$

and consequently $(\mathrm{Q})$ holds, when taking $B=A-A-A, C=-A$.

$(\mathrm{Q}) \Rightarrow(\mathrm{P})$ : Let $V$ be the closed convex hull of $B$ (which also is bounded). Then (2.1) implies (1.3), and by Theorem 1 we have the existence of an additive $a: S \rightarrow E$ such that $a(x)-f(x) \in V(x \in S)$. We get $r(x):=f(x)-a(x) \in$ $(-V)(x \in S)$, and it is sufficient to show that

$$
r(x) \in A \quad(x \in S) .
$$

Condition (2.2) implies for $x \in S$ that

$$
2^{k} r(x)-r\left(x^{2^{k}}\right) \in 2^{k} A+C,
$$

whence

$$
r(x) \in A+\frac{1}{2^{k}} C-\frac{1}{2^{k}} V,
$$

and $k \rightarrow \infty$ leads to $(2.3)$.

Remark 1 . Theorem 2 obviously characterizes perturbations of additive functions, where the perturbing function $r: S \rightarrow E$ has values in a given bounded closed subset $A$ of the Banach space $E$. In the special case (1.2) and

$$
A=\{\xi \mid \xi \in E,\|\xi\| \leq \varepsilon\} \quad(\text { where } \varepsilon>0),
$$

Theorem 2 is known from [10].

Remark 2. Let $S$ be a real or complex vector space, consider a Banach space $E$ having the same scalar field, and suppose $A$ to be a bounded closed subset of $E$. Then, similarly to Theorem 2, functions $f=L+r: S \rightarrow E$ can be characterized, where $L: S \rightarrow E$ is linear and $r(x) \in A(x \in S)$; cf. Haifeng Ma (马海 凤) and Volkmann [7]. In the special case of real spaces $S, E$, and $A$ as in (2.4), this characterization is known from Roman Ger and Volkmann [3]. 


\section{Some Tabor groupoids and the group $S_{3}$}

Let $S^{1}, S^{2}$ be Tabor groupoids and consider $S^{1} \times S^{2}$ equipped with the product $(x, y)(\bar{x}, \bar{y})=(x \bar{x}, y \bar{y})$ (for $\left.(x, y),(\bar{x}, \bar{y}) \in S^{1} \times S^{2}\right)$. Then $S^{1} \times S^{2}$ also is a Tabor groupoid, provided that the product in at least one of $S^{1}, S^{2}$ is square-symmetric, i.e., for $S=S^{1}$ or $S=S^{2}$ we have (1.2). If in addition ( $\left.\tilde{\mathrm{T}}\right)$ holds for $S^{1}, S^{2}$, then this condition also holds for $S^{1} \times S^{2}$.

Now we consider semigroups, that means groupoids, where the multiplication is associative. Every commutative semigroup $S$ obviously is a Tabor groupoid, in fact (1.2) holds. In the next Theorem we require the existence of an idempotent element, consequently groups are admissible.

Theorem 3. Let $S$ be a semigroup containing an element $s=s^{2}$. Then $S$ is a Tabor groupoid, if one of the following two conditions holds:

I) For every $x \in S$ there exists $n \in N_{0}$ such that $x^{2^{n}}=s$.

II) For every $x \in S$ there exists an odd $m \in N$ such that $x^{m}=s$.

Proof. Case I): Let us consider $x, y \in S$. There are $l, m, n \in N_{0}$ such that

$$
x^{2^{l}}=y^{2^{m}}=(x y)^{2^{n}}=s .
$$

Then $k:=l+m+n+1 \in N$ and (1.1) holds.

Case II): Let us consider $x, y \in S$. There are odd $m_{1}, m_{2}, m_{3} \in N$ such that $x^{m_{1}}=y^{m_{2}}=(y x)^{m_{3}}=s$. Then $m=m_{1} m_{2} m_{3}$ also is odd and

$$
x^{m}=y^{m}=(y x)^{m}=s .
$$

The number 2 being prime to $m$, it follows from Fermat's Theorem (cf., e.g., Harriet Griffin [6], p. 88) that $m$ divides $2^{\varphi(m)}-1$, where $\varphi$ denotes Euler's function. For $k=\varphi(m) \in N$ we thus have the existence of an $n \in N$ such that

$$
2^{k}-1=m n \text {. }
$$

Now (3.1) implies

$$
(y x)^{2^{k}-1}=(y x)^{m n}=s=s s=x^{m n} y^{m n}=x^{2^{k}-1} y^{2^{k}-1} .
$$

When multiplying this equation by $x$ from the left and by $y$ from the right, we finally get $(x y)^{2^{k}}=x^{2^{k}} y^{2^{k}}$.

Remark 3 . The semigroups treated in Theorem 3 also satisfy condition $(\tilde{\mathrm{T}})$.

The group $\boldsymbol{S}_{\mathbf{3}}$. We shall show that this group is not a Tabor groupoid; concerning the definition and basic properties of $S_{3}$ cf., e.g., Lidiâ Ivanovna Golovina [5], pp. 262, 263. Here we describe $S_{3}$ as the set of all bijections $\omega:\{1,2,3\} \rightarrow$ $\{1,2,3\}$, where the product is the composition of functions. Elements $\omega$ of $S_{3}$ are given in the form $\omega=(\omega(1) \omega(2) \omega(3))$; then $\iota=\left(\begin{array}{lll}1 & 2 & 3\end{array}\right)$ is the unit. Let us consider three elements of $S_{3}$ :

$$
\sigma=\left(\begin{array}{lll}
2 & 3 & 1
\end{array}\right), \quad \tau_{2}=\left(\begin{array}{lll}
3 & 2 & 1
\end{array}\right), \quad \tau_{3}=\left(\begin{array}{lll}
2 & 1 & 3
\end{array}\right) .
$$


We have

$$
\sigma=\sigma^{4}=\sigma^{16}=\sigma^{64}=\ldots, \quad \iota \neq \sigma^{2}=\sigma^{8}=\sigma^{32}=\sigma^{128}=\ldots,
$$

hence

$$
\sigma^{2^{n}} \neq \iota \quad\left(n \in N_{0}\right)
$$

Furthermore,

$$
\tau_{2}^{2}=\tau_{3}^{2}=\iota, \quad \sigma \tau_{3}=\tau_{2} .
$$

If $S_{3}$ is supposed to be a Tabor groupoid, then there would be a number $k \in N$ such that $\left(\sigma \tau_{3}\right)^{2^{k}}=\sigma^{2^{k}} \tau_{3}^{2^{k}}$. Taking (3.3) into account, we get $\iota=\sigma^{2^{k}}$, which is in contradiction with $(3.2)$.

\section{A stability result}

Lemma. Let $S$ be a groupoid satisfying $(\tilde{\mathrm{T}})$. When $x, y \in S$ are fixed, then (1.5) holds for infinitely many $k \in N$.

Proof. Let $k \in N$ be such that (1.5) holds. Condition ( $\tilde{\mathrm{T}})$ applied to $x, y$ replaced by $x^{2^{k}}, y^{2^{k}}$, respectively, yields the existence of $l \in N$ such that

$$
\left(x^{2^{k}} y^{2^{k}}\right)^{2^{l}}=x^{2^{k+l}} y^{2^{k+l}}, \quad\left(\left(x^{2^{k}} y^{2^{k}}\right) y^{2^{k}}\right)^{2^{l}}=\left(x^{2^{k+l}} y^{2^{k+l}}\right) y^{2^{k+l}} .
$$

Now it is sufficient to show that (1.5) holds with $k$ replaced by the greater number $k+l \in N$. Indeed, from (1.5) and (4.1) we get

$$
(x y)^{2^{k+l}}=\left((x y)^{2^{k}}\right)^{2^{l}}=\left(x^{2^{k}} y^{2^{k}}\right)^{2^{l}}=x^{2^{k+l}} y^{2^{k+l}}
$$

and

$$
((x y) y)^{2^{k+l}}=\left(((x y) y)^{2^{k}}\right)^{2^{l}}=\left(\left(x^{2^{k}} y^{2^{k}}\right) y^{2^{k}}\right)^{2^{l}}=\left(x^{2^{k+l}} y^{2^{k+l}}\right) y^{2^{k+l}} .
$$

Theorem 4. Let $S$ be a groupoid having a left unit and satisfying condition $(\tilde{\mathrm{T}})$. For $f: S \rightarrow R$ suppose that

$$
|\max \{f((x y) y), f(x)\}-f(x y)-f(y)| \leq \varepsilon \quad(x, y \in S) .
$$

Then there exists $g: S \rightarrow R$ such that

$$
\max \{g((x y) y), g(x)\}=g(x y)+g(y) \quad(x, y \in S)
$$

and

$$
|f(x)-g(x)| \leq 3 \varepsilon \quad(x \in S)
$$


Proof. As in [4] it can be shown that

$$
\left|f\left(x^{2}\right)-2 f(x)\right| \leq 3 \varepsilon \quad(x \in S) .
$$

For this proof, $S$ can be an arbitrary groupoid with a left unit. From (4.5) we get by [11] (for arbitrary groupoids $S$ ) the existence of

$$
g(x)=\lim _{n \rightarrow \infty} \frac{1}{2^{n}} f\left(x^{2^{n}}\right) \quad(x \in S)
$$

and the validity of (4.4). It is sufficient to verify (4.3): Let us fix $x, y \in S$. When replacing in (4.2) the elements $x, y$ by $x^{2^{k}}, y^{2^{k}}$, so that (1.5) holds, then we have

$$
\left|\max \left\{f\left(((x y) y)^{2^{k}}\right), f\left(x^{2^{k}}\right)\right\}-f\left((x y)^{2^{k}}\right)-f\left(y^{2^{k}}\right)\right| \leq \varepsilon .
$$

According to the preceding Lemma this inequality is true for infinitely many $k \in N$, which can be arranged in a sequence

$$
k_{1}<k_{2}<k_{3}<\ldots \rightarrow \infty \text {. }
$$

Now we consider (4.7) for all $k=k_{m}(m \in N)$, divide both sides by $2^{k_{m}}$ and let $m$ tend to infinity. According to (4.6) we get (4.3).

Remark 4. Under the assumptions of Theorem 4 it is routine to see that there exists only one solution $g: S \rightarrow R$ of the functional equation (4.3) for which the left-hand side of (4.4) is bounded.

\section{Acknowledgements}

We cordially thank Mrs. Marion Ewald for her typewriting of this paper. The research of the second author was supported by University of Silesia, Katowice (Selected Topics in the Theory of Functional Equations).

Open Access. This article is distributed under the terms of the Creative Commons Attribution License which permits any use, distribution, and reproduction in any medium, provided the original author(s) and the source are credited.

\section{References}

[1] Badora, R., Przebieracz, B., Volkmann, P.: Stability of the Pexider functional equation. Ann. Math. Silesianae 24(2010), 7-13 (2011)

[2] Badora, R., Przebieracz, B., Volkmann, P.: Stability of the functional equation $f(x y)=$ $f(y x)$ on groups, Remark at the Eleventh Katowice-Debrecen Winter Seminar on Functional Equations and Inequalities, Wisła-Malinka 2011. Ann. Math. Silesianae 25(2011), $116(2012)$

[3] Ger, R., Volkmann, P.: On sums of linear and bounded mappings. Abh. Math. Sem. Univ. Hamburg 68, 103-108 (1998) 
[4] Gilányi, A., Nagatou, K., Volkmann, P.: Stability of a functional equation coming from the characterization of the absolute value of additive functions. Ann. Funct. Anal. 1(2), 1-6 (2011). http://www.emis.de/journals/AFA

[5] Golovina, L.I.: Linejnaâ algebra i nekotorye ee priloženiâ. Nauka, Moskva (1971)

[6] Griffin, H.: Elementary theory of numbers. McGraw-Hill, New York (1954)

[7] Ma, H., Volkmann, P.: On bounded perturbations of linear operators. EVA STAR (2012), 5 pp. http://digbib.ubka.uni-karlsruhe.de/volltexte/1000028750

[8] Simon (Chaljub-Simon), A., Volkmann, P.: Caractérisation du module d'une fonction additive à l'aide d'une équation fonctionnelle. Aequationes Math. 47, 60-68 (1994)

[9] Tabor, J.: Remark 18, 22nd International Symposium on Functional Equations, Oberwolfach 1984. Aequationes Math. 29, 96 (1985)

[10] Volkmann, P.: On the stability of the Cauchy equation. In: Proceedings of the Numbers, Functions, Equations '98 International Conference, edited by Zsolt Páles, Janus Pannonius Tudományegyetem Pécs, pp. 150-151 (1998)

[11] Volkmann, P.: O stabilności równań funkcyjnych o jednej zmiennej. Sem. LV No. 11 (2001), 6 pp., Errata ibid. No. 11bis (2003), 1 p. http://www.math.us.edu.pl/smdk

Roman Badora, Barbara Przebieracz and Peter Volkmann

Instytut Matematyki

Uniwersytet Śląski

Bankowa 14

40-007 Katowice

Poland

e-mail: robadora@math.us.edu.pl; barbara.przebieracz@us.edu.pl

Peter Volkmann

Institut für Analysis

KIT

76128 Karlsruhe

Germany

Received: November 8, 2012 